\section{(6) OPEN ACCESS}

\title{
Semaphorin 4D inhibits neutrophil activation and is involved in the pathogenesis of neutrophil-mediated autoimmune vasculitis
}

\author{
Masayuki Nishide, 1,2,3 Satoshi Nojima, 2,3,4 Daisuke Ito, 2,3,5 Hyota Takamatsu, 1,3,2 \\ Shohei Koyama, ${ }^{1,3,2}$ Sujin Kang, ${ }^{2,3,6}$ Tetsuya Kimura, ${ }^{1,3,2}$ Keiko Morimoto, ${ }^{1,3,2}$ \\ Takashi Hosokawa, 1,3,2 Yoshitomo Hayama, 1,3,2 Yuhei Kinehara, 1,3,2 \\ Yasuhiro Kato, ${ }^{1,3,2}$ Takeshi Nakatani, ${ }^{1,3,2}$ Yoshimitsu Nakanishi, ${ }^{1,3,2}$ Takeshi Tsuda, ${ }^{2,3,7}$ \\ Jeong Hoon Park, ${ }^{2}$ Toru Hirano, ${ }^{1}$ Yoshihito Shima, ${ }^{1}$ Masashi Narazaki, ${ }^{1}$ Eiichi Morii, ${ }^{4}$ \\ Atsushi Kumanogoh ${ }^{1,3,2}$
}

\begin{abstract}
- Additional material is published online only. To view, please visit the journal online (http://dx.doi.org/10.1136/ annrheumdis-2016-210706).

For numbered affiliations see end of article.

\section{Correspondence to Assistant Professor Satoshi Nojima, Department of PathologyOsaka University Graduate School of MedicineSuita City, OsakaJapan; s_nojima@molpath.med.osaka- u.ac.jp and Professor Atsushi Kumanogoh, Department of Respiratory Medicine, Allergy and Rheumatic Diseases, Suita City 565-0871, Osaka, Japan; kumanogo@imed3.med.osaka- u.ac.jp}

Received 21 October 2016 Revised 14 March 2017 Accepted 14 March 2017 Published Online First 12 April 2017

\section{ABSTRACT}

Objectives Inappropriate activation of neutrophils plays a pathological role in antineutrophil cytoplasmic antibody (ANCA)-associated vasculitis (AAV). The aim of this study was to investigate the functions of semaphorin 4D (SEMA4D) in regulation of neutrophil activation, and its involvement in AAV pathogenesis.

Methods Serum levels of soluble SEMA4D were evaluated by ELISA. Blood cell-surface expression of membrane SEMA4D was evaluated by flow cytometry. To determine the functional interactions between neutrophil membrane SEMA4D and endothelial plexin B2, wildtype and SEMA4D ${ }^{-1-}$ mice neutrophils were cultured with an endothelial cell line (MS1) stained with SYTOX green, and subjected to neutrophil extracellular trap (NET) formation assays. The efficacy of treating human neutrophils with recombinant plexin B2 was assessed by measuring the kinetic oxidative burst and NET formation assays.

Results Serum levels of soluble SEMA4D were elevated in patients with AAV and correlated with disease activity scores. Cell-surface expression of SEMA4D was downregulated in neutrophils from patients with AAV, a consequence of proteolytic cleavage of membrane SEMA4D. Soluble SEMA4D exerted pro-inflammatory effects on endothelial cells. Membranous SEMA4D on neutrophils bound to plexin B2 on endothelial cells, and this interaction decreased NET formation. Recombinant plexin B2 suppressed neutrophil Rac1 activation through SEMA4D's intracellular domain, and inhibited pathogeninduced or ANCA-induced oxidative burst and NET formation.

Conclusions Neutrophil surface SEMA4D functions as a negative regulator of neutrophil activation. Proteolytic cleavage of SEMA4D as observed in patients with AAV may amplify neutrophil-mediated inflammatory responses. SEMA4D is a promising biomarker and potential therapeutic target for AAV.

\section{INTRODUCTION}

Neutrophils are the initial effectors of the innate immune response during acute inflammation. ${ }^{1}$ They play key roles in the clearance of extracellular pathogens through their production of reactive oxygen species (ROS), phagocytosis and subsequent intracellular killing, ${ }^{2}$ and the release of neutrophil extracellular traps (NET). ${ }^{34}$ These rapid and strong responses are tightly regulated. In peripheral blood vessels, vascular endothelial cells are thought to be important local modulators, directly contacting and interacting with neutrophils. ${ }^{5}$ However, the physiological mechanisms for this regulation are not fully understood.

Antineutrophil cytoplasmic antibody (ANCA)-associated vasculitis (AAV) is one of the severe and life-threatening autoimmune diseases. Inappropriate inflammatory response from neutrophils and their interaction with small vessel endothelium play important pathological roles in $\mathrm{AAV}^{6}{ }^{6}$ ROS production and NET formation are increased in patients with AAV, and enhanced formation and disordered regulation of NET can cause ANCA production and direct endothelial injury. ${ }^{8} 9$ AAV affects small vessels, where neutrophils are in close contact with the vascular endothelium. Therefore, the interaction between neutrophils and the endothelial cell surface is likely to be the initial and primary event in the pathogenesis of AAV, and the disruption of neutrophil-endothelium regulation during inflammation is thought to be an important factor. ${ }^{679}$

Semaphorins were originally identified as repulsive and collapsing neural guidance factors during neuronal development, ${ }^{10}$ and cumulative findings have demonstrated that these proteins have multiple roles for regulating immune responses. ${ }^{11}$ Semaphorin 4D (SEMA4D) was the first semaphorin shown to have a role in the immune system. ${ }^{12}$ SEMA4D, a transmembrane protein that also exists in a functional soluble form following proteolytic cleavage after cellular activation, binds several receptors, plexins B1/B2 and CD72, which affect immune responses. ${ }^{11}$ In addition, SEMA4D has been shown to have activities on vascular endothelial cells ${ }^{13}$ and neurons. ${ }^{14}$ Interestingly, SEMA4D works as a ligand and as a direct signalling receptor of plexin B2. ${ }^{15}$ Regarding the pathogenesis of autoimmunity, it has been reported that SEMA4D is involved in the development of experimental autoimmune encephalomyelitis (EAE), a model of 
multiple sclerosis, ${ }^{16}$ and rheumatoid arthritis. ${ }^{17}$ However, the functional roles and pathological implications of SEMA4D in neutrophils and neutrophil-mediated autoimmunity have not been clarified. In this study, we investigate the role of SEMA4D in regulation of neutrophil activation, and its involvement in the pathogenesis of AAV.

\section{METHODS}

\section{Human subjects}

All human samples were obtained after informed consent was provided by the subjects, in accordance with the Declaration of Helsinki and with approval from the ethical review board of the Graduate School of Medicine, Osaka University, Japan (no. 10237). This study was registered in the University Hospital Medical Information Network Clinical Trials Registry (UMIN000013076). Microscopic polyangiitis (MPA) and granulomatosis with polyangiitis (GPA) were defined according to 2012 Chapel Hill Consensus Conference nomenclature and definitions. Patients with AAV were diagnosed as having MPA or GPA, according to the criteria of the Research Committees of Japanese Ministry of Health, Labour and Welfare. GPA was also diagnosed according to the American College of Rheumatology classification criteria. Disease activity score of AAV was estimated by Birmingham Vasculitis Activity Score (BVAS), and AAV remission is defined as a BVAS score of 0 . Blood samples were also obtained from healthy individuals (HC) recruited from university staff.

\section{Human cell preparations}

Human neutrophils and peripheral blood mononuclear cells from healthy donors were separated by using Polymorphprep (Axis Shield). The samples were confirmed to consist of $>95 \%$ neutrophils by Wright-Giemsa staining. For some experiments, especially for separating neutrophils from patients, neutrophils were separated by using MACSxpress human neutrophil isolation kit (Miltenyi Biotec).

\section{Mouse cell preparations}

Mouse bone marrow neutrophils (from C57BL/6 mice) were isolated using MACS mouse neutrophil isolation kit (Miltenyi Biotec). The samples were confirmed to consist of $>95 \% \mathrm{Gr}-1^{+}$ $\mathrm{CD}_{11} \mathrm{~b}^{+}$neutrophils by flow cytometry on a FACS Canto II (BD Biosciences).

\section{Enzyme-linked immunosorbent assays}

Soluble SEMA4D levels in serum samples and cell culture supernatants were measured using an ELISA kit (Cloud-Clone). Serum samples were stored at $-80^{\circ} \mathrm{C}$ prior to ELISA. The detection range of SEMA4D was $1.56-100 \mathrm{ng} / \mathrm{mL}$. The concentrations of human ADAM17 in serum samples were determined using DuoSet ELISA kit (R\&D Systems). The detection range of ADAM17 was $156-10000 \mathrm{pg} / \mathrm{mL}$.

\section{Detection and quantitation of NET}

Neutrophils were seeded onto 12 -well plates at a density of $1 \times 10^{5}$ cells per well, with or without MS1. Cells were incubated for 1 hour in a $\mathrm{CO}_{2}$ incubator at $37^{\circ} \mathrm{C}$ to allow settling, and then stimulated with lipopolysaccharide (LPS) for indicated times. After stimulation, each well was carefully washed with phosphate-buffered saline, then SYTOX green (Invitrogen) was added to non-fixed live cells so that only extracellular DNA would be detected. Fluorescence images were acquired on a BZ-X700 microscope (Keyence). Quantitative analysis of NET formation was performed using 'Hybrid cell count system' in BZ-X700, an algorithm for accurate quantitation for images of cultured cells. Human NET formation was quantitated using Cayman's NET Assay Kit (Cayman Chemical).

\section{Time course monitoring of generated ROS}

ROS were measured by hydrogen peroxide fluorescence method, using the Amplite Fluorimetric Hydrogen Peroxide Assay Kit (AAT Bioquest). Human neutrophils were seeded onto 96-well plates at a density of $1 \times 10^{5}$ cells per well and were incubated for 1 hour in a $\mathrm{CO}_{2}$ incubator at $37^{\circ} \mathrm{C}$ to allow settling. Amplite substrates were added, and cells were subsequently stimulated with indicated stimuli for 2 hours. ROS levels were monitored using a fluorescence microplate reader (Infinite M1000) at Ex/ $\mathrm{Em}=540 / 590$ every $1 \mathrm{~min}$ for 120 cycles.

\section{Rac1 pull-down assay}

Rac1 pull-down assay was performed using the Active Rac1 Detection Kit (Cell Signaling). Briefly, cells were lysed, and GST-PAK1-PBD fusion protein was used to bind the activated form of GTP-bound Rac1, which was then immunoprecipitated with glutathione resin. Rac1 activation levels were then determined by western blotting with antihuman Rac1 antibody.

\section{IgG purification from human sera}

Serum IgG purification from $\mathrm{HC}$ or patients with AAV was performed using the Melon gel IgG purification kit (Pierce).

\section{Statistical analysis}

Non-parametric Mann-Whitney U test or a two-tailed unpaired Student's t-test was used to compare two groups. Data are expressed as means $\pm S D$ or means \pm SEM. $p$ values $<0.05$ were considered significant. *p<0.05; **p $<0.01 ; * * \mathrm{p}<0.001$. Correlations between clinical parameters and serum soluble SEMA4D levels were determined using Spearman's rank correlation coefficient $(\rho)$.

\section{RESULTS}

\section{Increased levels of soluble SEMA4D in patients with AAV}

In search for molecules that regulate neutrophil activation, we found the expression of semaphorins, SEMA3A, SEMA4D and $S E M A 7 A$, in both naïve mouse bone marrow neutrophils and peritoneal exudate neutrophils (see online supplementary figure S1). Interestingly, the expression of SEMA4D was significantly different between these types of neutrophils. Therefore, we first investigated the profile of SEMA4D in clinical patients suffering from diseases affiliated with neutrophil disorders.

Serum concentrations of soluble SEMA4D were measured in healthy donors $(n=16)$, in patients with bacterial infections (BI) $(n=29)$, and in patients with $\operatorname{AAV}(n=33)$. Clinical data and demographics were presented in online supplementary table S1. Serum soluble SEMA4D levels were significantly higher in patients with AAV than in healthy donors or patients with BI (figure 1A). Serum soluble SEMA4D levels were positively correlated with vasculitis activity score (Birmingham Vasculitis Activity Score 2008 version 3) and with blood neutrophil counts, whereas there were no apparent correlations between soluble SEMA4D levels and C reactive protein (CRP) levels or MPO-ANCA titres (figure 1B). Flow cytometry revealed that cell-surface expression of SEMA4D was significantly downregulated in polymorphonuclear cells from patients with AAV compared with healthy donors. By contrast, cell-surface 
A

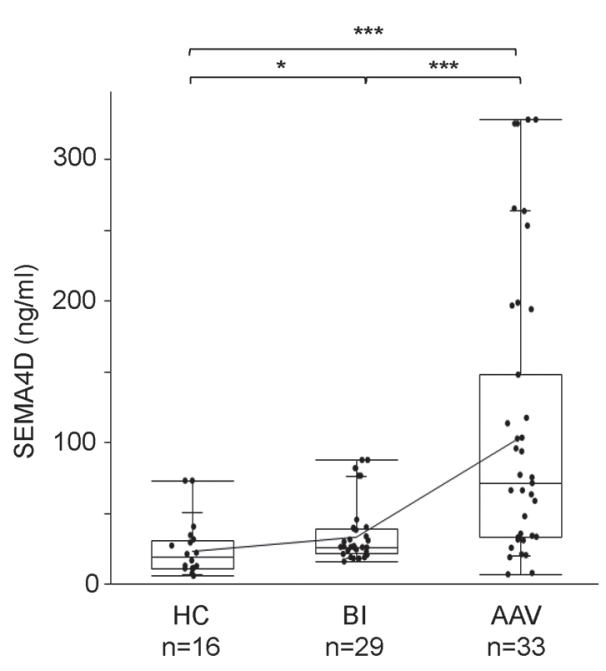

B
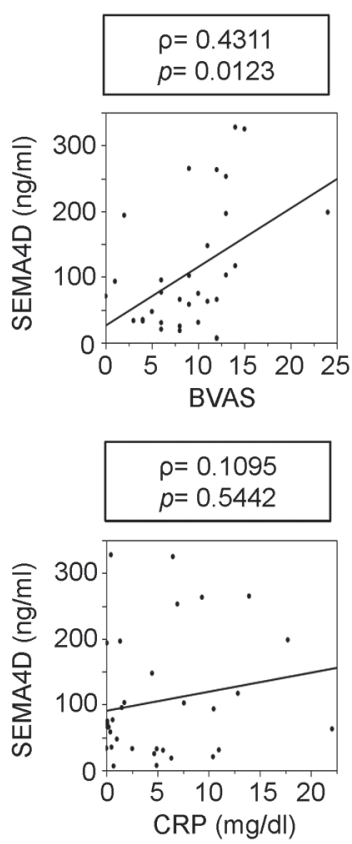

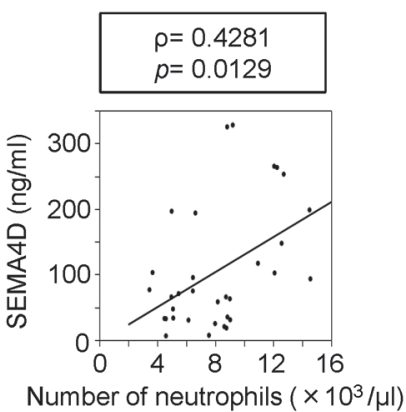

Number of neutrophils $\left(\times 10^{3} / \mu \mathrm{l}\right)$

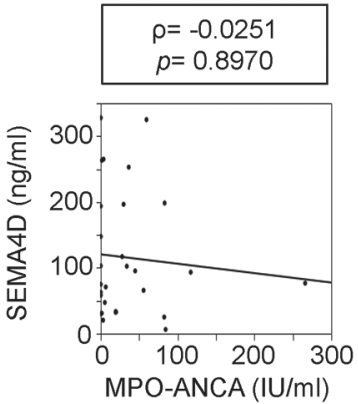

C

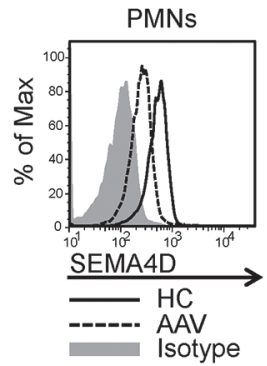

D

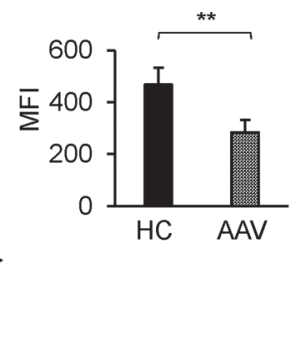

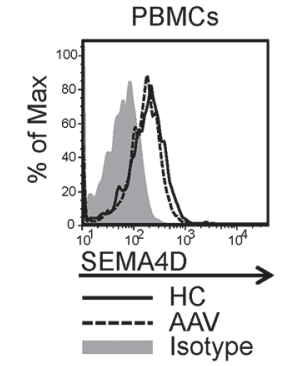

Figure 1 Identification of semaphorin 4D (SEMA4D) as a neutrophil activation marker associated with ANCA-associated vasculitis (AAV).

(A) Soluble SEMA4D levels in serum samples from 16 healthy individuals (HC), 29 patients with bacterial infections (BI) and 33 patients with AAV.

(B) Correlation of soluble SEMA4D serum levels with Birmingham Vasculitis Activity Score (BVAS) 2008 version 3, blood neutrophil counts, C reactive protein (CRP) levels and MPO-ANCA titres. Correlations were quantified using Spearman's rank correlation coefficient ( $\rho$ ). Flow cytometry of SEMA4D cell-surface expression by peripheral blood polymorphonuclear cells (PMNs) (C) and mononuclear cells (PBMCs) (D) from healthy individuals (HC; solid line) and patients with AAV (AAV; dotted line). Cells from HC were also stained with an isotype-matched control antibody (isotype; grey-filled histogram). Mean fluorescence intensity (MFI) profiles were determined from five HC or five patients with AAV. NS, not significant ( $p>0.05$ ); ${ }^{*} p<0.05$; ${ }^{* *} \mathrm{p}<0.01 ;{ }^{* *} \mathrm{p}<0.001$, as determined by a non-parametric Mann-Whitney $\mathrm{U}$ test (A) or a two-tailed unpaired Student's t-test (C-D). Values are means \pm SD $(A)$ and means \pm SEM $(C-D)$.

expression of SEMA4D was not changed in the blood mononuclear cells from patients with AAV (figure 1C, D).

\section{Shedding of SEMA4D in stimulated neutrophils}

We hypothesised that the increased levels of soluble SEMA4D and the decreased levels of membrane-bound SEMA4D on neutrophils in patients with AAV were due to the shedding of SEMA4D from the activated neutrophil cell surface, likely from other immune cell types. ${ }^{18} 19$ To verify this, isolated neutrophils derived from a healthy donor were incubated with N-formyl-methionyl-leucyl-phenylalanine (FMLP). After stimulation, cell-surface expression of SEMA4D was significantly decreased and the levels of soluble SEMA4D in the culture supernatant were accordingly increased (figure 2A). These results indicate that cell-surface SEMA4D is proteolytically cleaved from stimulated neutrophils to yield a soluble form. SEMA4D can be cleaved by several metalloproteinases. ${ }^{18}{ }^{20}$ We therefore investigated enzymatic activity for neutrophil SEMA4D cleavage with matrix metalloprotease (MMP)-8, MMP-9 and tumour necrosis factor (TNF)- $\alpha$ converting enzyme (TACE/ADAM17), which are involved in neutrophil-mediated immune responses ${ }^{21}$ and functionally activated on the FMLP-stimulated neutrophils. ${ }^{22-24}$ ADAM17 decreased expression of SEMA4D on the neutrophil cell surface (figure 2B) and significantly induced the release of soluble SEMA4D into the culture supernatant (figure 2C). ADAMTS4, a reported sheddase for monocyte membranous SEMA4D,${ }^{17}$ did not influence neutrophil SEMA4D shedding (see online supplementary figure S2). To further confirm that ADAM17 is involved in the cleavage of neutrophil cell-surface SEMA4D, we pretreated neutrophils with Tapi-1, a potent ADAM17 inhibitor, then stimulated them with FMLP. As expected, the cleavage of SEMA4D in these cells was abrogated (figure 2D). Importantly, a previous report has shown that serum concentrations of circulating ADAM17 were increased in patients with $\mathrm{AAV}^{25}$ Consistent with this, we detected elevated levels of serum ADAM17 in patients with AAV (figure 2E). Thus, 
A
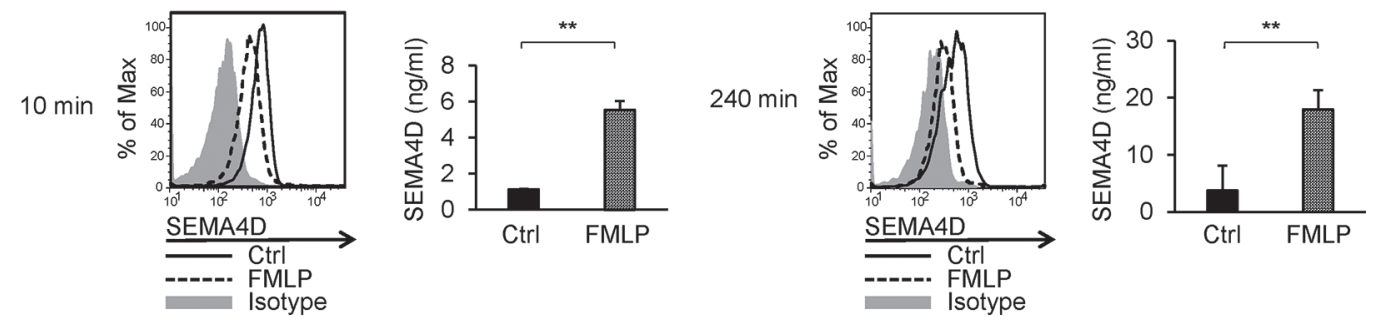

B
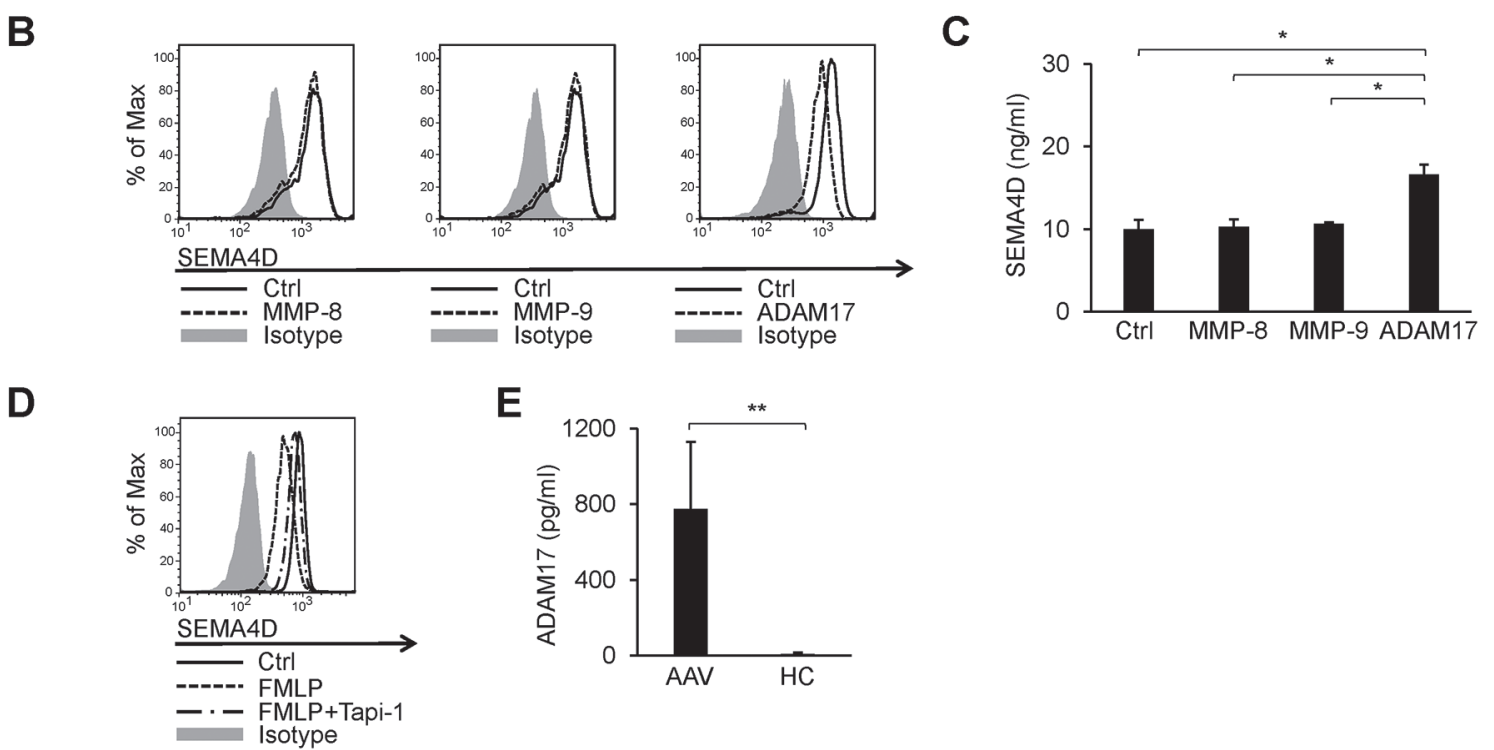

Figure 2 Shedding of semaphorin 4D (SEMA4D) in stimulated neutrophils. (A) Cell-surface expression of SEMA4D on isolated human neutrophils derived from healthy donors, stimulated with or without formyl-methionyl-leucyl-phenylalanine (FMLP). Flow cytometry was performed at the indicated time points (10 or $240 \mathrm{~min}$ ) on neutrophils cultured with control medium containing $2 \mu \mathrm{M}$ FMLP (dotted line) or control medium (Ctrl; solid line). Cells from the control medium were also stained with an isotype-matched control antibody (isotype; grey-filled histogram). Soluble SEMA4D levels in the culture supernatants at each time point were determined by ELISA. (B) SEMA4D cell-surface expression on isolated human neutrophils stimulated with metalloproteases. Isolated neutrophils were primed with tumour necrosis factor (TNF)- $\alpha$ ( $2 \mathrm{ng} / \mathrm{mL}$ ) for $30 \mathrm{~min}$, and then stimulated either recombinant matrix metalloprotease (MMP)-8 (5 $\mu \mathrm{g} / \mathrm{mL})$, MMP-9 $(5 \mu \mathrm{g} / \mathrm{mL})$ or TNF- $\alpha$ converting enzyme (ADAM17; $5 \mu \mathrm{g} / \mathrm{mL})$ for $60 \mathrm{~min}$. Flow cytometry was performed similarly to (A). Soluble SEMA4D levels in each culture supernatant were determined by ELISA (C). (D) Effects of ADAM17 inhibitor Tapi-1 on cell-surface cleavage of SEMA4D. Isolated human neutrophils were pretreated with Tapi-1 (40 $\mu$ M) for 30 min, followed by stimulation with FMLP $(2 \mu \mathrm{M})$ for $30 \mathrm{~min}$. Flow cytometry was performed similarly to (A). (E) Serum levels of ADAM17 in 33 patients with ANCAassociated vasculitis (AAV) and 16 healthy individuals (HC). NS, not significant $(p>0.05) ;{ }^{*} p<0.05 ;{ }^{*} p<0.01 ;{ }^{* * *} p<0.001$, as determined by a two-tailed unpaired Student's t-test $(A, C)$ or a non-parametric Mann-Whitney $U$ test $(E)$. Values are means $\pm S E M(A, C)$ and means $\pm S D(E)$. Data are representative of three independent experiments $(A-D)$.

these data suggest that SEMA4D is proteolytically cleaved from stimulated neutrophils to yield a soluble form, a process for which ADAM17 is relevant.

\section{Assessment of soluble SEMA4D functions on endothelium}

Previous reports suggested that soluble SEMA4D exerts pro-inflammatory effects on endothelium. ${ }^{132627}$ Consistent with this, we confirmed that soluble SEMA4D promoted the permeability (see online supplementary figure S3A) and interleukin (IL)-8 secretion of endothelial cells (see online supplementary figure S3B), without affecting the expression of endothelial adhesion molecules (see online supplementary figure S3C). To further assess the significance of SEMA4D in AAV pathogenesis, we next studied an in vivo neutrophil-mediated vasculitis model, ${ }^{28}$ using wild-type (WT) and SEMA4D-deficient $\left(S E M A 4 D^{-/-}\right)$mice. Contrary to expectations, SEMA4D ${ }^{-/-}$mice exhibited enhanced phenotypes in comparison with WT mice (see online supplementary figure S4A-E). These findings prompted us to hypothesise that, in addition to the functions of soluble SEMA4D, reduction of neutrophil membrane-bound SEMA4D might be pathologically important in AAV.

Impaired NET formation in SEMA4D ${ }^{-/-}$neutrophils in contact with endothelial cells

To investigate the functions of membrane-bound SEMA4D on neutrophils, we compared several functions in neutrophils derived from WT and SEMA4D ${ }^{-1-}$ mice. SEMA4D was expressed in both bone marrow-derived and peripheral bloodneutrophils (see online supplementary figure S5A). Cell-surface SEMA4D expression was significantly reduced in peritoneal exudate neutrophils, similar to human SEMA4D expressed on stimulated neutrophils (see online supplementary figure S5B). Notably, there were no significant differences in in vivo peritoneal migration, in vitro viability, adherent capacity, surface expression of adhesion molecules between neutrophils derived from WT and SEMA4D ${ }^{-/-}$mice (see online supplementary figure S5C-F). Enhanced formation of NET is an important 
A

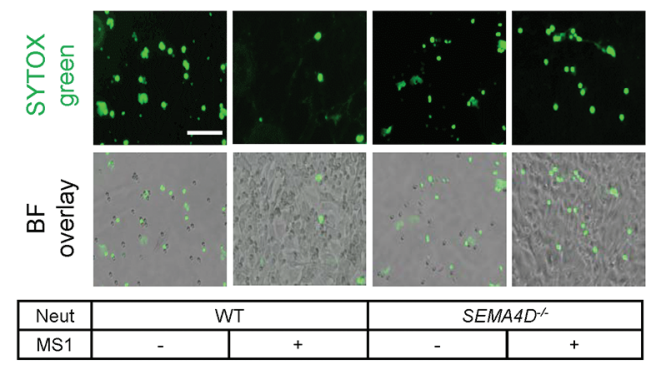

c
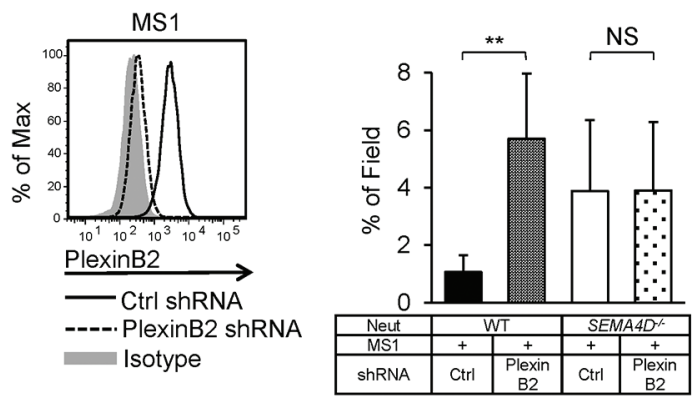

B

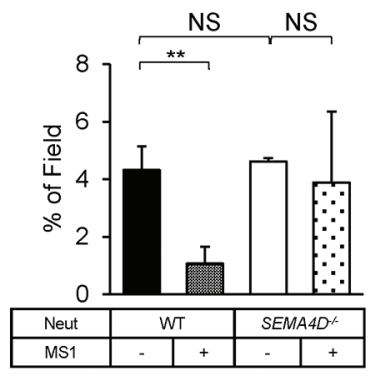

E
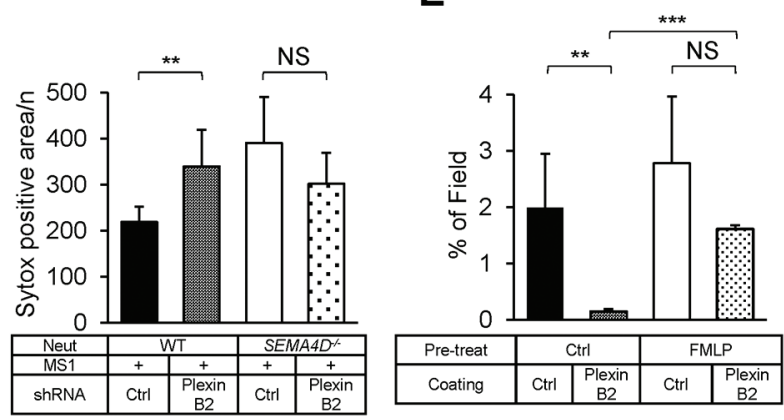

Figure 3 Interaction between neutrophil semaphorin 4D (SEMA4D) and endothelial plexin B2 negatively regulates neutrophil extracellular trap (NET) formation. (A) SYTOX green immunofluorescence analysis was performed on bone marrow-derived neutrophils isolated from wild-type (WT) or SEMA4D-deficient $\left(S E M A 4 D^{-l-}\right.$ ) mice cocultured with or without MS1 cells and treated with lipopolysaccharide (LPS) $(10 \mu \mathrm{g} / \mathrm{mL})$ for $4 \mathrm{hours}$ to induce NET formation. Scale bar: $100 \mu \mathrm{m}$. (B) Quantification of NET formation. Data are expressed as total SYTOX green-positive extracellular DNA area $\left(\mu \mathrm{m}^{2}\right)$ per field (over 10 randomly chosen fields in each experiment) and average SYTOX green-positive area ( $\left.\mu \mathrm{m}^{2}\right)$ was normalised against the total number of SYTOX-positive neutrophils in the same fields (400-500 neutrophils per field were counted and averaged in each experiment). (C) Knockdown of plexin B2 in MS1 cells. Cell-surface expression of plexin B2 was analysed using flow cytometry. MS1 cells treated with control short hairpin RNA (shRNA) were also stained with the isotype-matched control antibody (isotype; grey-filled histogram). (D) Effects of plexin B2 knockdown in MS1 cells on NET formation. Quantification of NET formation was performed similarly to (B). (E) Effects of plexin B2 on human NET formation. Isolated neutrophils derived from healthy donors were stimulated with or without $2 \mu \mathrm{M} \mathrm{FMLP,} \mathrm{washed} \mathrm{and} \mathrm{subsequently} \mathrm{seeded} \mathrm{onto} \mathrm{culture} \mathrm{plates}$ precoated with recombinant human plexin B2 protein or control his-tag protein. These cells were incubated for 60 min, then stimulated with LPS (10 $\mu \mathrm{g} / \mathrm{mL}$ ) for 4 hours. SYTOX green immunofluorescence analysis was performed and evaluated similarly to (A and B). NS, not significant ( $p>0.05)$; ${ }^{*} p<0.05 ;{ }^{* *} p<0.01 ;{ }^{* *} p<0.001$, as determined by a two-tailed unpaired Student's t-test. Values are means $\pm S E M(B, D, E)$. Data are representative of three independent experiments $(A-E)$.

factor which leads to ANCA production and endothelial injury. ${ }^{9}$ We thus examined whether a SEMA4D defect in neutrophils influences NET formation. Bone marrow neutrophils isolated from WT or SEMA4D ${ }^{-/-}$mice were cultured with and without an endothelial cell line, MS1, and stained with SYTOX green (figure 3A). SYTOX green is a cell-impermeable DNA-binding dye, so its fluorescence intensity in non-fixed cultures is indicated NET formation. ${ }^{29}$ NET formation of WT neutrophils attached to MS1 was significantly reduced. By contrast, NET formation from SEMA4D ${ }^{-1-}$ neutrophils were not (figure 3A, B). These findings indicate cellular contact between WT neutrophils and endothelial cells negatively regulates NET formation. In addition, SEMA4D seemed to function as a receptor that suppresses neutrophil activation via interaction with a molecule expressed on endothelial cells. As previously reported, SEMA4D functions as a receptor for plexin B2 in activated gamma-delta T cells. ${ }^{15}$ We therefore focused on endothelial plexin B2 as a functional ligand of neutrophil SEMA4D receptor. Plexin B2 was expressed on MS1 cells and on human umbilical vein endothelial cells (see online supplementary figure S6A). By contrast, plexin B2 was not expressed on neutrophils (see online supplementary figure S6B). When neutrophils were attached to MS1 cells transfected with control short hairpin RNA (shRNA), NET formation was significantly suppressed. By contrast, when neutrophils were attached to MS1 cells in which plexin B2 was knocked down by shRNA (figure 3C), this suppressive effect was inhibited (figure 3D and online supplementary figure S7A). Of note, the adhesion capacities of WT and SEMA4D ${ }^{-1-}$ neutrophils were almost the same (see online supplementary figure S7B). Thus, these data indicate that the interactions between endothelial plexin B2 ligand and neutrophil SEMA4D receptor inhibit NET formation, and this regulatory function cannot be replaced by other semaphorin-plexin interactions. To confirm these findings, we stimulated healthy human neutrophils with LPS on culture dishes precoated with recombinant plexin B2. Supporting our previous findings, NET formation by plexin B2-treated neutrophils was significantly suppressed (see online supplementary figure S8), and FMLP-induced shedding of neutrophil SEMA4D weakened this protective effect (figure 3E).

Regulation of ROS production by the SEMA4D-plexin B2 interaction ROS production is upstream of NET formation ${ }^{4}$ and is correlated with NET formation in neutrophils from patients with AAV. ${ }^{8}$ Therefore, we evaluated ROS production by plexin B2-treated neutrophils. Production of ROS after treatment with LPS (figure 4A; image of subsequent NET formation from each culture was attached) and other stimuli (figure 4B) was 
A

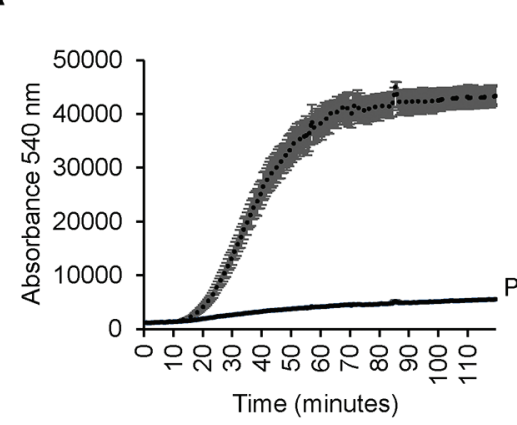

B

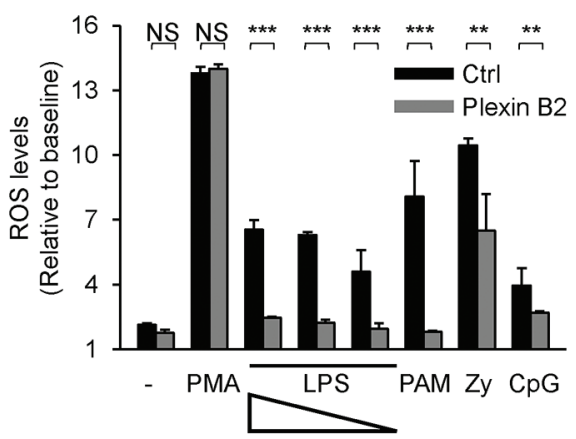

$\mathbf{E}$
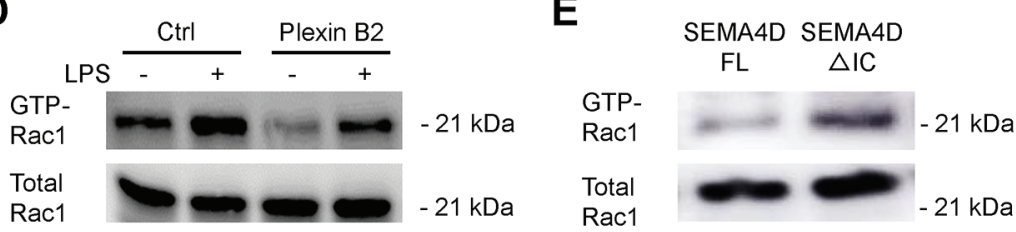

G
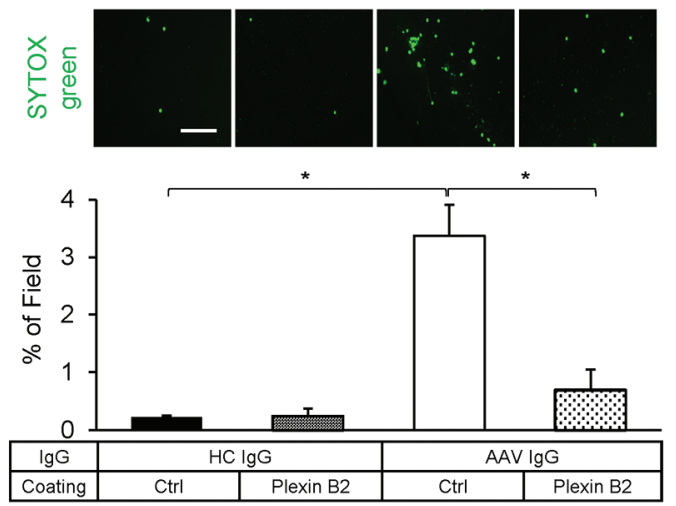

H

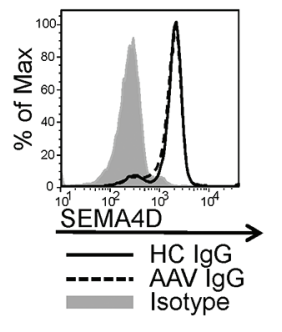

Figure 4 Inhibition of the neutrophil oxidative burst by the semaphorin 4D (SEMA4D)-plexin B2 interaction. (A) Effects of plexin B2 on the generation of reactive oxygen species (ROS) in human neutrophils. Neutrophils isolated from healthy donors were seeded onto culture plates precoated with recombinant human plexin B2 or control his-tag protein. These cells were incubated for 60 min, then stimulated with lipopolysaccharide (LPS) $(1 \mu \mathrm{g} / \mathrm{mL}$ ) for 2 hours. ROS levels were monitored every 1 min for a total of 120 cycles, and representative traces are the means from one experiment performed in triplicate. SYTOX green immunofluorescence analysis was performed at the last period of 4 hours after LPS stimulation, and image of neutrophil extracellular trap (NET) formation from each culture was attached. Scale bar: $100 \mu \mathrm{m}$. (B) Effects of plexin B2 on the generation of ROS in response to various stimulants. Isolated neutrophils were seeded similarly to (A) and were either left unstimulated (-) or stimulated with Phorbol 12-myristate 13-acetate (PMA) $(1 \mu \mathrm{M}), \mathrm{LPS}(100 \mu \mathrm{g} / \mathrm{mL}, 1 \mu \mathrm{g} / \mathrm{mL}$ and $100 \mathrm{ng} / \mathrm{mL})$, Pam3CSK4 (1 $\mu \mathrm{g} / \mathrm{mL}), \mathrm{Zymosan}(1 \mu \mathrm{g} /$ $\mathrm{mL}$ ) or CpG-ODN (1 $\mu \mathrm{g} / \mathrm{mL})$ for 2 hours. Relative ROS levels were determined as the ratio of fluorescent peak to baseline. (C) Dose-dependent effects of plexin B2 on ROS generation. Isolated neutrophils were seeded and stimulated similarly to (A), on culture plates precoated with recombinant human plexin B2 at the indicated concentrations. (D) Effects of plexin B2 on Rac1 activation in human neutrophils. Isolated neutrophils were seeded and incubated for $60 \mathrm{~min}$ similarly to (A) (LPS-), then stimulated with LPS (1 $\mu \mathrm{g} / \mathrm{mL})$ for $20 \mathrm{~min}$ (LPS+). Cells were lysed at each time point, and GTP-bound Rac1 was pulled down using GST-PAK-CRIB as described. Proteins were separated by SDS-PAGE $\left(5 \times 10^{6}\right.$ cells/lane) and blotted with antihuman Rac1 antibody. (E) GTP-bound Rac1 pull-down assay using SEMA4D-transfected TLR4-expressing HEK293 cells. TLR4-expressing HEK293 cells were transfected with a construct expressing either full-length SEMA4D (FL) or SEMA4D without the intracellular C-terminal domain ( $\triangle \mathrm{IC})$. Subsequently, cells were stimulated with LPS (1 $\mu \mathrm{g} / \mathrm{mL} ; 40 \mathrm{~min}$ ) and lysed, then GTP-bound Rac1 was pulled down and blotted similarly to (D). Effects of plexin B2 on ROS production (F) and NET formation $(G)$ in response to purified IgG from a healthy donor (HC $\lg G$ ) or a patient with AAV (AAV lgG). Isolated neutrophils were primed with tumour necrosis factor (TNF)- $\alpha$ ( $2 \mathrm{ng} / \mathrm{mL}$ ) for $15 \mathrm{~min}$, then seeded similarly to (A), and stimulated with each immunoglobulin $(200 \mu \mathrm{g} / \mathrm{mL}$ ) for 1 hour (ROS) or 4 hours (NET). NET formation was evaluated similarly to (figure 3B). Scale bar: $200 \mu \mathrm{m}$. (H) Flow cytometry of cell-surface SEMA4D on neutrophils, primed and stimulated similarly to (F). NS, not significant $(p>0.05) ;{ }^{*} p<0.05$; ${ }^{* *} p<0.01$; ${ }^{* * *} p<0.001$, as determined by a two-tailed unpaired Student's t-test. Values are means \pm SEM $(A-C, F$ and $G)$. Data are representative of three independent experiments $(\mathrm{A}-\mathrm{H})$.

significantly suppressed by plexin B2; the inhibition ratio was dependent on the concentration of precoated plexin B2 protein (figure 4C) and FMLP-induced neutrophil SEMA4D shedding weakened this inhibitory effect (see online supplementary figure
S9A). Furthermore, in the case of mouse neutrophils, in an in vivo zymosan-induced peritonitis model, ROS production was suppressed by plexin B2 treatment in WT peritoneal neutrophils, whereas ROS production in SEMA4D ${ }^{-/-}$mice was not 
suppressed by plexin B2 treatment (see online supplementary figure S9B). These data support that the SEMA4D-plexin B2 interaction is required for the suppression of ROS production, as well as NET formation. To determine the mechanism of this suppression, we evaluated the LPS-mediated signalling pathway in detail. The expression of neutrophil surface TLR4, neutrophil membrane polarisation and the phosphorylation of MAP kinases were not different between control and plexin B2-treated neutrophils (see online supplementary figure S10A-C). However, the activation of Rho-family small GTPase Rac1, which is essential for neutrophil ROS production, ${ }^{30}$ was strikingly inhibited in plexin B2-treated neutrophils (figure 4D and online supplementary figure 11). Furthermore, HEK293 cells expressing TLR4 that were transfected with a construct expressing fulllength SEMA4D had a significant reduction of Rac1 activation in response to LPS stimulation, whereas cells transfected with a construct expressing SEMA4D without its intracellular C-terminal domain did not show such reduction (figure 4E). These results suggest that plexin B2-SEMA4D binding inhibits ROS production by suppressing Rac1 activity through SEMA4D's intracellular domain.

\section{Regulation of ANCA IgG-induced ROS production by plexin B2 treatment}

Finally, we examined the ability of recombinant plexin B2 to regulate excessive ROS production and NET formation in patients with AAV. IgG purified from patients with AAV can induce greater ROS production and NET formation than IgG from healthy individuals. ${ }^{831}$ We purified serum IgG from healthy donors (HC IgG) or patients with AAV (AAV IgG, derived from patient no. 12 in online supplementary table 1), and evaluated ROS production and NET formation following stimulation with these immunoglobulins. As expected, AAV IgG induced more ROS production and NET formation, and treatment of neutrophils with plexin B2 suppressed ROS production and NET formation to levels comparable to those in cells stimulated with HC IgG (figure 4F, G). Of note, these immunoglobulins did not influence SEMA4D expression on neutrophils (figure $4 \mathrm{H}$ ). Taken together, our data show that SEMA4D has pro-inflammatory functions on endothelial cells and inhibits neutrophil activation by acting as a receptor for endothelial plexin B2. Alteration of the SEMA4D-plexin B2 interaction by proteolytic cleavage of membranous SEMA4D is involved in the pathogenesis of AAV (figure 5).

\section{DISCUSSION}

Here, we identified the novel regulatory mechanisms of neutrophils through neutrophil-endothelium interactions to maintain immunological homeostasis and highlighted the pathological importance of SEMA4D-plexin B2 axis in AAV, providing basic and clinical implications.

Serum levels of SEMA4D were significantly elevated in patients with AAV and positively correlated with the disease activity score (figure 1A, B). SEMA4D levels in patients with AAV were much higher than in patients with other autoimmune diseases, such as rheumatoid arthritis or systemic lupus erythematosus, which we reported previously. ${ }^{17}$ In addition, cell-surface expression of SEMA4D was significantly decreased in neutrophils, but not in any other leucocytes from patients with AAV (figure 1C, D). In current strategies for management for vasculitis, serum CRP levels are considered to be useful as a clinical marker. However, several studies have demonstrated that CRP concentrations do not always correlate with disease activity or relapse probability of $\mathrm{AAV}^{32}$ Our findings suggest the possibility of SEMA4D as an additional clinical marker for the prediction of AAV disease activity and for the evaluation of neutrophil activation status.

\section{Healthy}

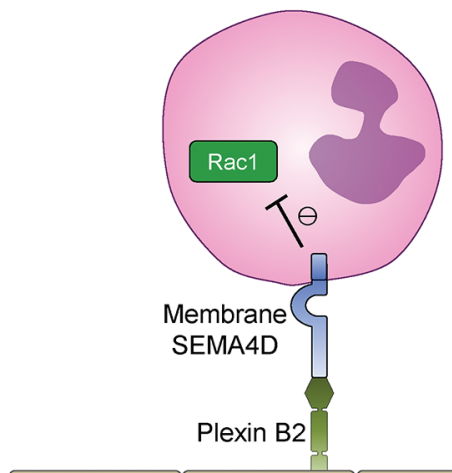

AAV

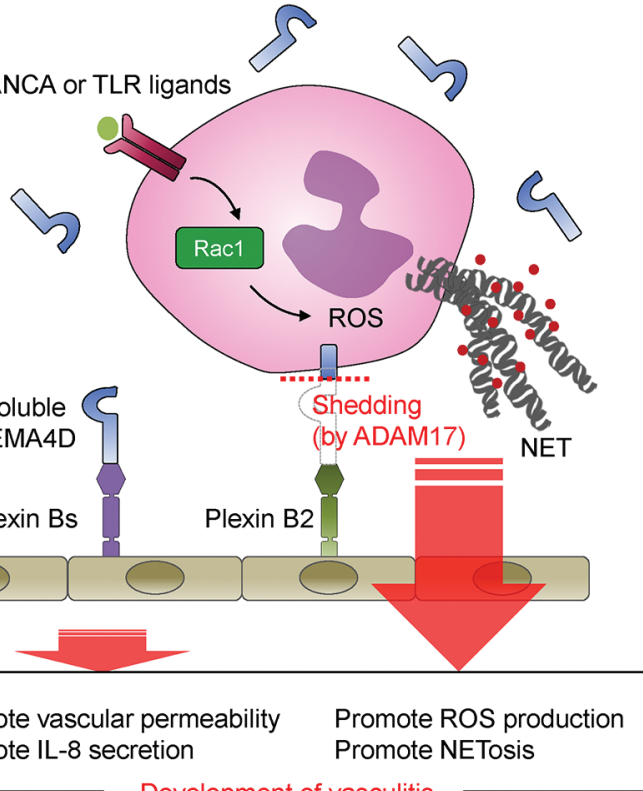

Figure 5 Graphical scheme of this study. Under healthy conditions, the interaction between endothelial plexin B2 ligand and neutrophil cells surface SEMA4D receptor inhibits Rac1 activation and negatively regulates the generation of ROS and NET formation. By contrast, in patients with AAV, neutrophil surface SEMA4D is shed by ADAM17. Soluble SEMA4D had pro-inflammatory functions on endothelial cells. In addition, alteration of the SEMA4D-plexin B2 interaction results in aberrant activation of neutrophils, and this dichotomous effect is involved in the pathogenesis of AAV. 
SEMA4D is a transmembrane protein that exists also in a soluble form following proteolytic cleavage on cellular activation. ${ }^{18}{ }^{19}$ Consistent with this, SEMA4D is proteolytically cleaved from stimulated neutrophil cell surface to yield a soluble form, a process for which ADAM17 is relevant (figure 2). Soluble SEMA4D increased the permeability and IL-8 secretion of endothelial cells. In addition, it has been demonstrated that SEMA4D promotes B-cell antibody production and induces growth cone collapse in the nerve system. ${ }^{11}$ Indeed, patients with AAV exhibit significant neutrophil infiltration around small vessels; elevated serum IL-8 and ANCA concentrations, resulting in neutrophil activation; and peripheral nerve disorders. In this regard, soluble SEMA4D may be an exacerbating factor on AAV. However, SEMA4D ${ }^{-/-}$mice showed severer phenotypes in the experimental vasculitis model (see online supplementary figure S4). The model which we performed was a classical immunecomplex-mediated glomerulonephritis rather than AAV models, but the generation of MPO-ANCA and neutrophil-mediated glomerular inflammation in this model have been demonstrated. ${ }^{28}$ Our in vivo findings support that the loss of membrane-bound SEMA4D is crucially involved in the development of neutrophil-mediated vascular inflammation.

We also revealed that a direct cellular interaction between neutrophils and endothelial cells negatively regulates NET formation in a SEMA4D-plexin B2-dependent manner (figure 3). Of note, a unique interaction between SEMA4D and plexin B2 has been previously reported. ${ }^{15}$ In their study, SEMA4D, which usually works as a ligand, functions as a receptor for plexin B2 ligand in activated gamma-delta $\mathrm{T}$ cells, and this interaction promotes wound healing. Our findings are consistent with their idea, that is, SEMA4D receptor binding would generate intracellular signalling in neutrophils.

Recombinant plexin B2 treatment could suppress the production of ROS (figure 4A, B), which occurs upstream of NET formation. ${ }^{4}$ Rac1 activation, which is involved in the production of ROS by promoting NADPH membrane translocation, was significantly inhibited in plexin B2-treated neutrophils through the intracellular domain of SEMA4D (figure 4D, E). According to previous reports, even when Rac1 activation is reduced slightly, ROS production is significantly suppressed. ${ }^{30}$ In our study, stronger inhibition of Rac1 activity was observed in repeated experiments. Previous studies have also reported that inhibition of Rac1 activity does not affect the cytokine production mediated by the MAP kinase signalling pathway. ${ }^{30}$ Accordingly, in our experiment, the SEMA4D-plexin B2 interaction suppressed Rac1 activity without affecting MAP kinase signalling. Furthermore, treatment of neutrophils with plexin $\mathrm{B} 2$ suppressed the induction of ROS production and NET formation by IgG from patients with AAV (figure 4F, G). Physicians currently rely on glucocorticoids and cytotoxic drugs such as cyclophosphamide for the treatment of $\mathrm{AAV}^{33} \mathrm{As}$ an alternative therapeutic option, membrane-bound SEMA4D represents a potential target for preventing the aberrant activation of neutrophils.

In summary, the neutrophil SEMA4D receptor inhibits inflammatory responses by interacting with the endothelial plexin B2 ligand. In small vessels where neutrophils are in close contact with endothelium, this interaction minimises inappropriate activation of neutrophils. Under conditions in which ADAM17 is functionally activated, such as AAV, membrane-bound SEMA4D is cleaved from the surface of neutrophils. Soluble SEMA4D had pro-inflammatory functions on endothelial cells. In addition, alteration of the SEMA4D-plexin B2 interaction results in aberrant activation of neutrophils, and this dichotomous effects are involved in the pathogenesis of AAV (figure 5). SEMA4D has potential as a serological marker of AAV, and represents a promising therapeutic target for clinical management of neutrophil-mediated autoimmune vasculitis.

\section{Author affiliations}

${ }^{1}$ Department of Respiratory Medicine and Clinical Immunology, Osaka University Graduate School of Medicine, Suita City, Osaka, Japan

${ }^{2}$ Laboratory of Immunopathology, World Premier International Immunology Frontier Research Center, Suita City, Osaka, Japan

${ }^{3}$ The Japan Agency for Medical Research and Development-Core Research for Evolutional Science and Technology (AMED-CREST), Japan

${ }^{4}$ Department of Pathology, Osaka University Graduate School of Medicine, Suita City, Osaka, Japan

${ }^{5}$ Department of Nephrology, Osaka University Graduate School of Medicine, Suita City, Osaka, Japan

${ }^{6}$ Department of Clinical Application of Biologics, Osaka University Graduate School of Medicine, Suita City, Osaka, Japan

${ }^{7}$ Department of Otorhinolaryngology-Head and Neck Surgery, Osaka University Graduate School of Medicine, Suita City, Osaka, Japan

Acknowledgements This work was supported by research grants from AMED-CREST and AMED (to AK); COI stream and SRIP grants from the Ministry of Education, Culture, Sports, Science and Technology (MEXT) of Japan (to AK); from the Ministry of Health, Labour and Welfare of Japan (to AK); from MEXT/JSPS KAKENHI Grant Number 15612282 (to SN) and from the Takeda Science Foundation (to SN) and the Ube Industries Foundation (to SN).

Contributors AK designed and supervised the project. MN carried out most of experiments. SN, DI, TT and YN conducted some of experiments. HT, SK, SK, TK, KM EM and AK contributed to preparation of materials and provided advice on project planning and data interpretation. MN, TH, YH, YK, YK, TN, TH, YS and MN recruited and clinically characterised patients. MN, SN, JP and AK wrote the manuscript. SN and AK provided funding for the study. All authors contributed to the discussion of the results and edited and approved the final version.

Competing interests None declared.

Patient consent All human samples were obtained after informed consent was provided by the subjects, in accordance with the Declaration of Helsinki and with approval from the ethical review board of the Graduate School of Medicine, Osaka University, Japan (no. 10237).

Ethics approval The ethical review board of the Graduate School of Medicine, Osaka University, Japan (no. 10237). This study was registered in the University Hospital Medical Information Network Clinical Trials Registry (UMIN000013076).

Provenance and peer review Not commissioned; externally peer reviewed.

Open Access This is an Open Access article distributed in accordance with the Creative Commons Attribution Non Commercial (CC BY-NC 4.0) license, which permits others to distribute, remix, adapt, build upon this work non-commercially, and license their derivative works on different terms, provided the original work is properly cited and the use is non-commercial. See: http://creativecommons.org/ licenses/by-nc/4.0/

(c) Article author(s) (or their employer(s) unless otherwise stated in the text of the article) 2017. All rights reserved. No commercial use is permitted unless otherwise expressly granted.

\section{REFERENCES}

1 Nauseef WM, Borregaard N. Neutrophils at work. Nat Immunol 2014;15:602-11.

2 Phillipson M, Kubes P. The neutrophil in vascular inflammation. Nat Med 2011;17:1381-90.

3 Brinkmann V, Reichard U, Goosmann C, et al. Neutrophil extracellular traps kill Bacteria. Science 2004;303:1532-5.

4 Brinkmann V, Zychlinsky A. Neutrophil extracellular traps: is immunity the second function of chromatin? J Cell Biol 2012;198:773-83.

5 Ji JY, Jing H, Diamond SL. Hemodynamic regulation of inflammation at the endothelial-neutrophil interface. Ann Biomed Eng 2008;36:586-95

$6 \mathrm{Hu} \mathrm{N}$, Westra J, Kallenberg CG. Dysregulated neutrophil--endothelial interaction in antineutrophil cytoplasmic autoantibody (ANCA)-associated vasculitides: implications for pathogenesis and disease intervention. Autoimmun Rev 2011;10:536-43.

7 Halbwachs L, Lesavre P. Endothelium-neutrophil interactions in ANCA-associated diseases. J Am Soc Nephrol 2012;23:1449-61.

8 Ohlsson SM, Ohlsson S, Söderberg D, et al. Neutrophils from vasculitis patients exhibit an increased propensity for activation by anti-neutrophil cytoplasmic antibodies. Clin Exp Immuno/ 2014;176:363-72.

9 Nakazawa D, Shida H, Tomaru U, et al. Enhanced formation and disordered regulation of NETs in myeloperoxidase-ANCA-associated microscopic polyangiitis. J Am Soc Nephrol 2014;25:990-7. 
10 Kolodkin AL. Semaphorins: mediators of repulsive growth cone guidance. Trends Cell Biol 1996;6:15-22.

11 Kumanogoh A, Kikutani H. Immunological functions of the neuropilins and plexins as receptors for semaphorins. Nat Rev Immunol 2013;13:802-14.

12 Bougeret C, Mansur IG, Dastot $H$, et al. Increased surface expression of a newly identified 150-kDa dimer early after human T lymphocyte activation. J Immunol 1992;148:318-23.

13 Sierra JR, Corso S, Caione L, et al. Tumor angiogenesis and progression are enhanced by Sema4D produced by tumor-associated macrophages. J Exp Med 2008;205:16731673-85.

14 Swiercz JM, Kuner R, Behrens J, et al. Plexin-B1 directly interacts with PDZ-RhoGEF/ LARG to regulate RhoA and growth cone morphology. Neuron 2002;35:51-63.

15 Witherden DA, Watanabe M, Garijo 0, et al. The CD100 receptor interacts with its plexin B2 ligand to regulate epidermal $\gamma \delta$ T cell function. Immunity 2012;37:314-25.

16 Okuno T, Nakatsuji Y, Moriya M, et al. Roles of Sema4D-plexin-B1 interactions in the central nervous system for pathogenesis of experimental autoimmune encephalomyelitis. J Immunol 2010;184:1499-506.

17 Yoshida Y, Ogata A, Kang S, et al. Semaphorin 4D contributes to rheumatoid arthritis by inducing inflammatory cytokine production: pathogenic and therapeutic implications. Arthritis Rheumato/ 2015;67:1481-90.

18 Elhabazi A, Delaire S, Bensussan A, et al. Biological activity of soluble CD100. I. the extracellular region of CD100 is released from the surface of T lymphocytes by regulated proteolysis. J Immunol 2001;166:4341-7.

19 Wang X, Kumanogoh A, Watanabe C, et al. Functional soluble CD100/Sema4D released from activated lymphocytes: possible role in normal and pathologic immune responses. Blood 2001;97:3498-504.

20 Zhu L, Bergmeier W, Wu J, et al. Regulated shedding of sema4D from the platelet surface produces a bioactive second messenger in thrombotic disorders. Blood 2005;106:1641.
21 Khokha R, Murthy A, Weiss A. Metalloproteinases and their natural inhibitors in inflammation and immunity. Nat Rev Immunol 2013;13:649-65.

22 Owen CA, Hu Z, Lopez-Otin C, et al. Membrane-bound matrix metalloproteinase-8 on activated polymorphonuclear cells is a potent, tissue inhibitor of metalloproteinase-resistant collagenase and serpinase. J Immunol 2004;172:7791-803.

23 Takafuji S, Ishida A, Miyakuni Y, et al. Matrix metalloproteinase-9 release from human leukocytes. J Investig Allergol Clin Immunol 2003;13:50-5.

24 Walcheck B, Herrera AH, St Hill C, et al. ADAM17 activity during human neutrophil activation and apoptosis. Eur J Immunol 2006;36:968-76.

25 Bertram A, Lovric S, Engel A, et al. Circulating ADAM17 level reflects disease activity in Proteinase-3 ANCA-Associated vasculitis. J Am Soc Nephrol 2015:26:2860-70.

26 Zhou H, Yang YH, Basile JR. The semaphorin 4D-Plexin-B1-RhoA signaling Axis recruits pericytes and regulates vascular permeability through endothelial production of PDGF-B and ANGPTL4. Angiogenesis 2014;17:261-74.

27 Yang $\mathrm{YH}$, Zhou H, Binmadi NO, et al. Plexin-B1 activates NF- $\mathrm{KB}$ and IL-8 to promote a pro-angiogenic response in endothelial cells. PLoS One 2011;6:e25826.

28 Yumura W, Itabashi M, Ishida-Okawara A, et al. A novel mouse model for MPO-ANCAassociated glomerulonephritis. Microbiol Immunol 2006;50:149-57.

29 Brinkmann V, Laube B, Abed UA, et al. Neutrophil extracellular traps: how to generate and visualize them. J Vis Exp 2010;24:pii1724.

30 Condliffe AM, Webb LM, Ferguson GJ, et al. RhoG regulates the neutrophil NADPH oxidase. J Immuno/ 2006;176:5314-20.

31 Jennette JC. Pathogenesis of ANCA vasculitis.. Curr Opin Nephrol Hypertens 2011:20:263-70.

32 Jennette JC, Falk RJ, Vasculitis S-VN Engl J Med 1997;337:1512-23.

33 Selamet U, Kovaliv YB, Savage CO, et al. ANCA-associated vasculitis: new options beyond steroids and cytotoxic drugs. Expert Opin Investig Drugs 2007;16:689-703. 